\title{
A proof-of-concept study on CGRP plasma levels of migraineurs during a 6-month treatment with ERENUMAB
}

\author{
Giuseppe Tringali', Catello Vollono ${ }^{2,3}$, Paolo Calabresi ${ }^{3,4}$ and Pierluigi Navarra ${ }^{1,5^{*}}$
}

\begin{abstract}
The introduction of monoclonal antibodies (mAbs) against calcitonin-gene related peptide (CGRP) or CGRP receptors in the treatment of migraine raised concerns on the possible risks associated to the long-term inhibition of CGRP physiological functions. In this proof-of-concept study, we have measured the circulating levels of CGRP in 7 patients with high-frequency episodic migraine receiving the anti-CGRP receptor mAb erenumab for at least 6 months, to test the hypothesis that long-term blockade of CGRP receptors induces an increase in systemic CGRP levels via a classical up-regulation mechanism.

Plasma CGRP levels were measured by a validated radioimmunoassay at baseline, and after 1 and 6 months of treatment with erenumab, 70 mg given sc every 4 weeks.

We found (data expressed as the means \pm SD): $38.34 \pm 30.74 \mathrm{pg} C \mathrm{GRP} / \mathrm{ml}$ of plasma at baseline, $38.19 \pm 29.23 \mathrm{pg} / \mathrm{ml}$ after 1 month and $53.89 \pm 28.03 \mathrm{pg} / \mathrm{ml}$ after 6 months of treatment. Thus, the average increase in plasma CGRP levels after 6 months of treatment was about $+40 \%$ compared to both baseline and 1-month treatments; such difference was not statistically significant because of high SD values in all groups.

These preliminary findings need to be confirmed in larger, sufficiently powered experiments.
\end{abstract}

Keywords: CGRP, CGRP receptor, Monoclonal antibodies, Erenumab, Migraine

In the June 2019 issue, the journal Peptides published our last work on calcitonin gene-related peptide (CGRP), a review dealing with the anti-CGRP and anti-CGRP receptor monoclonal antibodies (mAbs) recently introduced for migraine treatment [1]. The paper addressed the concerns raised by the potential risks ensuing a long-term inhibition of CGRP functions, and we discussed whether the different action mechanisms of these mAbs (i.e. quenching systemic CGRP vs blocking its receptors) might be associated to different safety profiles.

\footnotetext{
*Correspondence: pierluigi.navarra@unicatt.it

'Department of Healthcare Surveillance and Bioethics, Section of

Pharmacology, Università Cattolica del Sacro Cuore, Largo F. Vito 1, 00168 Rome, Italy

${ }^{5}$ Pharmacology - Fondazione Policlinico Universitario Agostino Gemelli IRCCS, Rome, Italy

Full list of author information is available at the end of the article
}

Among other issues, we made a point of measuring plasma CGRP levels during long-term treatments with anti-CGRP mABs [1]. Concerns about the potential risks associated to long-term blockade of CGRP or CGRP receptor are shared by several groups [2-5], whereas a more optimistic view foresees a remarkable safety profile, based on the concept that anti-CGRP and antiCGRP receptor mAbs knockdown, but do not knockout CGRP signaling [6].

First-in-class $\mathrm{mAb}$, the anti-CGRP receptor erenumab obtained a marketing authorization in the European Union on July 26, 2018 (https://www.ema.europa.eu/en/ medicines/human/EPAR/aimovig), but only very recently the price negotiation process has been completed in Italy (https://www.gazzettaufficiale.it/eli/gu/2020/07/21/182/ $\mathrm{sg} / \mathrm{pdf})$. During the time that erenumab was not 
commercially available, the drug has been provided through an expanded access program, and a limited number of patients have been treated at our Headache Clinics, 'Fondazione Policlinico Gemelli IRCCS' Academic Hospital in Rome.

Having in mind the risk hypotheses postulated in our review [1], we used a previously validated CGRP radioimmunoassay [7] to measure the levels of circulating free CGRP in the plasma of patients included in the expanded access program of our Center, and treated with erenumab at approved dosages for at least 6 months. Seven patients with high-frequency episodic migraine, meeting the criteria of erenumab labeling, had a complete set of blood samples collected: all the patients received the 70-mg dose. All patients provided an informed consent for use of biological samples, according to the rules of Gemelli Hospital, and the study protocol was approved by the Independent Ethics Committee of the hospital.

We found (data expressed as pg CGRP/ml of plasma, the means \pm SD of 7 replicates per group): $38.34 \pm 30.74$ at baseline; $38.19 \pm 29.23$ after 1 month of treatment (i.e. after a single administration); $53.89 \pm 28.03$ after 6 months of treatment (i.e. at steady-state). Thus, the average increase in circulating CGRP after 6 months of erenumab treatment was about $+40 \%$ compared to both baseline and 1-month treatments, although such difference was not statistically significant because of huge SDs in all groups.

We are fully aware of the limitations of this study. The number of patients included is small, and overall variability is subsequently high. Assuming a SD around $30 \mathrm{pg} / \mathrm{ml}$, for a size effect of $+40 \%$ to be statistically significant, a sample size of at 55 subject is required, with a power of $80 \%$ and a risk of type-I error $<5 \%$ in a two-tail test. Nonetheless, we believe it may be useful to publish this proof-of-concept study, in order to stimulate discussion on the issue of the biological effects of this new class of drugs, and possibly to prompt further, adequately powered studies on this topic. Such patho-physiological investigations might usefully complement the clinical studies that are currently under way to establish the effectiveness of erenumab in the real-world setting [8].

\section{Acknowledgements}

Not applicable.

\section{Authors' contributions}

GT carried out the assays and analyzed the data. CV followed up the patients and collected the blood samples. PC reviewed and edited the paper. PN conceived the study, analyzed the data, and wrote the original draft. The authors read and approved the final manuscript.

\section{Funding}

This work received no financial support. The study drug was available through an expanded access program, as stated in the main text.
Ethics approval and consent to participate

All patients provided an informed consent for use of biological samples, according to the rules of Gemelli Hospital (this statement is also included in the main text).

\section{Consent for publication}

All authors reviewed the final version of the manuscript and provided their consent for publication.

\section{Competing interests}

None to declare.

\section{Author details}

'Department of Healthcare Surveillance and Bioethics, Section of Pharmacology, Università Cattolica del Sacro Cuore, Largo F. Vito 1, 00168 Rome, Italy. ${ }^{2}$ Neurofisiopatologia - Fondazione Policlinico Universitario Agostino Gemelli IRCCS, Rome, Italy. ${ }^{3}$ Neurologia - Università Cattolica del Sacro Cuore, Rome, Italy. ${ }^{4}$ Neurologia - Fondazione Policlinico Universitario Agostino Gemelli IRCCS, Rome, Italy. ${ }^{5}$ Pharmacology - Fondazione Policlinico Universitario Agostino Gemelli IRCCS, Rome, Italy.

Received: 1 September 2020 Accepted: 16 October 2020

Published online: 21 October 2020

\section{References}

1. Tringali G, Navarra P (2019) Anti-CGRP and anti-CGRP receptor monoclonal antibodies as antimigraine agents. Potential differences in safety profile postulated on a pathophysiological basis. Peptides 116:16-21

2. Deen M, Correnti E, Kamm K, Kelderman T, Papetti L, Rubio-Beltran E, Vigneri S, Edvinsson L (2017) Maassen Van Den brink a, on behalf of the European headache Federation School of Advanced Studies (EHF-SAS), blocking CGRP in migraine patients - a review of pros and cons. J Headache Pain 18:96

3. Giamberardino MA, Affaitati G, Costantini R, Cipollone F, Martelletti P (2017) Calcitonin gene-related peptide receptor as a novel target for the management of people with episodic migraine: current evidence and safety profile of erenumab. J Pain Res 10:2751-2760

4. MaassenVanDenBrink A, Meijer J, Villalon CM, Ferrari MD (2016) Wiping out CGRP: potential cardiovascular risks. Trends Pharmacol Sci 37:779-788

5. Rivera-Mancilla E, Villalón CM, MaassenVanDenBrink A (2020) CGRP inhibitors for migraine prophylaxis: a safety review. Expert Opin Drug Saf 21:1-14

6. Russo AF (2019) CGRP-based migraine therapeutics: how might they work, why so safe, and what next? ACS Pharmacol Transl Sci 2:2-8

7. Capuano A, Currò D, Dello Russo C, Tringali G, Pozzoli G, Di Trapani G, Navarra P (2007) Nociceptin (1-13) NH2 inhibits stimulated calcitonin-generelated-peptide release from primary cultures of rat trigeminal ganglia neurones. Cephalalgia 27:868-876

8. Lambru G, Hill B, Murphy M, Tylova I, Andreou AP (2020) A prospective real-world analysis of erenumab in refractory chronic migraine. J Headache Pain 21:61

\section{Publisher's Note}

Springer Nature remains neutral with regard to jurisdictional claims in published maps and institutional affiliations. 